\title{
Classification and Capture of Work-Related Non-Fatal Injuries Through a Real-Time Syndromic Surveillance System
}

\author{
Marija Borjan* and Margaret Lumia \\ New Jersey Department of Health, Trenton, NJ, USA
}

\section{Objective}

The New Jersey Department of Health (NJDOH), Occupational Health Surveillance (OHS) Unit staff proposes to evaluate a realtime surveillance tool to track a variety of occupationally-related emergency room visits throughout the state via EpiCenter, the NJDOH's existing real-time surveillance system.

\section{Introduction}

Syndromic surveillance has been used by state agencies to collect real-time information on disease outbreaks but has not been used to collect data in the occupational setting. Therefore, OHS staff has begun evaluating a real-time surveillance tool to track a variety of occupationally-related emergency room visits throughout the state via EpiCenter, the NJDOH's existing real-time surveillance system. This proposal applies established epidemiologic techniques to a different set of circumstances than they have been applied to in the past. Incorporating Syndromic Surveillance data with hospital discharge data will enhance the ability to classify and capture work-related nonfatal injuries and improve efforts of prevention. By employing a realtime, independent data source such as EpiCenter, the classification of work-related injuries and illnesses could be greatly enhanced, leading to a better understanding of the burden of non-fatal work-related injuries and illnesses, and allowing for quicker intervention.

\section{Methods}

A work-related injuries and illness classifier will be developed to trigger EpiCenter to alert staff of an occupational event. Classifiers are specific to certain events such as, heat related illness or chemical exposure; are composed of keywords related to those events; and are searchable in the chief complaint fields. Once an initial draft classification is developed, the historic data will be surveyed for the defined keywords or International Classification of Diseases (ICD) codes to refine the inclusion/exclusion keywords. Using a modified SAS code, keywords and ICD codes will be pulled from the historic data set. Once the syndrome classification is developed and validated, preliminary alert thresholds for work-related injury or illness events based on counts of Emergency Department (ED) events which meet the occupational syndromes will be determined. These alert thresholds may be based on absolute number of events or based on the number of cases needed for NJDOH Public Employee Occupational Health and Safety (PEOSH) to respond to. OHS staff will conduct sensitivity analysis to identify threshold cut-offs and long-term surveillance to detect trends in injuries.

\section{Results}

OHS currently receives automatic real-time electronic notifications when three or more cases of chemical exposures are seen in the same ED or among residents in the same county in NJ, within a 24hour period. OHS staff quickly reviews the cases using the secure EpiCenter website and contacts the ED Nurse Manager to obtain information on the chemical the patients were exposed too, how they were exposed, and if it was work-related. Recently, OHS staff began exploring using EpiCenter as a real-time surveillance system for occupationally related chemical exposures. This real-time surveillance of occupational events has detected diverse exposures and illnesses including: exposure to toluene vapor at a nail polish manufacturing plant; six cases of pesticide exposure at a produce repacking facility; and carbon monoxide poisoning of two police officers due to exhaust problems in their vehicle.

\section{Conclusions}

This use of the EpiCenter chief complaint reporting system has shown that it can yield real-time knowledge of incidents and local conditions for OHS to assist with and identify prevention opportunities. Occupational surveillance currently involves collecting data on potential cases on a quarterly or yearly basis, often long after the diagnosis was made. However, EpiCenter allows staff to identify illnesses early so a rapid response can be initiated, reducing further risk of occupational injuries and illness. The use of multiple data sources can help identify populations, occupations and industries at high risk of a work-place injury and illness in real-time, along with providing data to help monitor trends of work-related injuries and illness over time.

\section{Keywords}

occupational; injuries; surveillance

\section{Acknowledgments}

This project was funded by NIOSH Cooperative Grant \#5U60OH00848508 .

\section{References}

1. Pransky G, Snyder T, Dembe A, Himmelstein, J. Under-reporting of work-related disorders in the workplace: A case study and review of the literature. Ergonomics. 1999; 42.

2. Rosenman KD, Kalush A, Reilly MJ, Gardiner JC, Reeves M, Luo Z. How much work-related injury and illness is missed by the current national surveillance system? Journal of Occupational and Environmental Medicine. 2006; 48

\section{*Marija Borjan}

E-mail: Marija.Borjan@doh.state.nj.us 